\title{
Time to positivity of blood culture is a risk factor for clinical outcomes in Staphylococcus aureus bacteremia children: a retrospective study
}

Yuanyuan $\mathrm{Li}^{1,2}$, Qinyuan $\mathrm{Li}^{1,2}$, Guangli Zhang ${ }^{3}$, Huan $\mathrm{Ma}^{1,2}, \mathrm{Yi}_{\mathrm{W}} \mathrm{Wu}^{1,2}$, Qian $\mathrm{Yi}^{1,2}$, Lili Jiang ${ }^{1,2}$, Jiao Wan ${ }^{1,2}$, Fengtao Suo ${ }^{1,2}$ and Zhengxiu Luo ${ }^{3^{*}}$ (i)

\begin{abstract}
Background: Staphylococcus aureus (S. aureus) is a common cause of bacteremia, which leads to significant morbidity and mortality. We investigated the relationship between time to positivity (TTP) and clinical outcomes in children with S.aureus bacteremia in the China.

Methods: A retrospective study of Staphylococcus aureus bacteremia inpatient was performed in Children's Hospital of Chongqing Medical University in China between 29 January 2014 and 29 August 2017. TTP and clinical parameters were determined and analyzed. The receiver operating characteristic (ROC) curves were plotted for optimal cut-off selection, multivariate logistic regression tests were performed to evaluate the association between TTP and clinical outcomes.

Results: Overall, 84 cases were enrolled. We stated that in-hospital mortality is significantly higher in the early TTP $(\leq 17 \mathrm{~h})$ than in the late TTP (> $17 \mathrm{~h}$ ) group $(57.14 \%$ vs $7.14 \%, P=0.000)$. Septic shock occurred in $57.14 \%$ of patients with early TTP and in $18.57 \%$ of patients with late TTP $(P=0.002)$. Detailed multivariate and statistical analysis revealed that early TTP, need for vasoactive agent were independent risk factors of in-hospital mortality; early TTP, need for vasoactive agent and APACHE $\|$ score $\geq 15$ were independent risk factors of septic shock incidence in S. aureus bacteremia children.
\end{abstract}

Conclusions: Overall, TTP of $\leq 17 \mathrm{~h}$ appeared to correlate with the worse outcomes for S. aureus bacteremia children. These results have important implications in the assessments and management of pediatric $S$. aureus bacteremia in a clinical setting.

Trial registration: Retrospectively registered.

Keywords: Time to positivity, S.aureus bacteremia, Children

\section{Background}

Staphylococcus aureus (S. aureus) is a common cause of bloodstream infections [1] and S. aureus bacteremia (SAB) leads to significant morbidity and mortality, both in adults and children [2-4]. The prevalence of SAB in the pediatric populations of industrialized nations is high, ranging from 8.4 to $30 / 100,000$ person-years $[5,6]$. Previous studies

\footnotetext{
* Correspondence: luozhengxiu816@hospital.cqmu.edu.cn

${ }^{3}$ Department of Respiratory Medicine, Children's Hospital of Chongqing

Medical University, Chongqing 401122, China

Full list of author information is available at the end of the article
}

emphasized the importance of early laboratory detection of SAB $[7,8]$, and demonstrated the time to positivity (TTP) of blood culture may serve as a predictor of clinical outcomes for adult SAB patients [9-12]. TTP has also been studied in children's bacteremia caused by $H$. influenza [13] , S. pneumonia [13, 14], and N. meningitides [15]. Several studies indicated that early TTP was a poor prognostic factor in adult SAB patients $[10,16,17]$. However, few studies have assessed the value of TTP in SAB children, the relationship between TTP and the clinical outcomes of SAB children remains unclear. Here we aim to evaluate the 
relationship between TTP and clinical presentations and outcomes, to explore the risk factors of in-hospital mortality, septic shock incidence in children with SAB.

\section{Methods}

\section{Study design and patients}

This study was performed in Children's Hospital of Chongqing Medical University, a 1500-bed tertiary level III teaching hospital in Chongqing, China. SAB children hospitalized in Children's Hospital of Chongqing Medical University between 29 January 2014 and 29 August 2017 were enrolled retrospectively. The inclusions had the following characteristics: (i) inpatients (not include the emergency department); (ii) age < 18 years; (iii) with $\geq 1 S$. aureus blood culture positive; (iv) with systemic inflammation reaction syndrome status. The exclusion criteria included any of the following: (i) patients who were lost to follow-up; (ii) patients with incomplete clinical information; (iii) patients without TTPs in medical records.

\section{Definitions}

Staphylococcus aureus bacteremia (SAB) was defined as at least 1 positive $S$. aureus blood culture with systemic inflammation reaction syndrome status (SIRS), SIRS was defined according to the criteria previous published on Pediatr Crit Care Med [18]. Time to positivity (TTP) was defined as the time period between blood incubation and the positive signal, whereby all individuals were included only once. When multiple cultures were positive, the shortest TTP was enrolled. Prior antibiotic exposure was considered when the antibiotic was used before hospital-admission, regardless of administration time and dosage, appropriateness was judged by latter susceptibility results. Immunosuppressants consisted of corticosteroid and other cytotoxic agents, it was considered when duration of use $\geq 1$ month. Neutropenia in this manuscript was associated with chemotherapy, defined by ANC (absolute neutrophil count) $<6.0 \times 10^{9} / \mathrm{L}$ (infants), $<1.0 \times 10^{9} / \mathrm{L}$ ( $<1$ year old), and $<1.5 \times 10^{9} / \mathrm{L}$ ( $\geq 1$ year old). The source of infection consisted of pneumonia, bone and joint, skin or soft tissue, endocarditis, CNS (central nervous system) infection, incisions, catheter related infection (urethral catheters and thoracic drain tube), and multiple sites. Nosocomial infection was defined as $S$. aureus infection that occurred $>48 \mathrm{~h}$ after hospital (not include the emergency department) admission or $<48 \mathrm{~h}$ after discharge. The length of hospitalization was defined as the time period between admission and discharge. The classification of septic shock was defined according to the criteria previously published in JAMA [19].

\section{Microbiologic method}

Approximately $3 \mathrm{~mL}$ of blood was inoculated into BACTEC plus aerobic bottles, which were then transported to the laboratory and immediately incubated in an automated continuous monitoring system. The BD diagnostic system was used for blood culture, which monitors $\mathrm{CO}_{2}$ production every $5 \mathrm{~min}$ by means of a fluorescent signal. Bottles with positive results were examined by Gram staining, and their contents were subcultured. Species identification and susceptibility tests were performed using Vitek identification and susceptibility cards (bioMe'rieux Vitek).

\section{Data collection}

The collected data included TTP of blood culture, demographic characteristics (age, gender, weight), underlying conditions (hematologic malignancy, congenital heart disease, neutropenia, primary immunodeficiency, chronic kidney disease), hypoalbuminemia, APACHE (acute physiology and chronic health evaluation) II scores, sources of infection as mentioned earlier, prior antibiotic exposure, appropriate antibiotic exposure, immunosuppressants use, nosocomial infection, methicillin-resistance, need for invasive mechanic ventilation, need for vasoactive agent, length of hospitalization, the incidence of septic shock and in-hospital mortality.

\section{Clinical outcomes}

The primary outcome was in-hospital mortality. Since previous studies suggested that septic shock meant worse outcomes in adult SAB patients [20], it was chosen as the second outcome.

\section{Statistical methods}

The continuous variables were presented as medians, and the first and third quartiles; the Student's $t$ test, Chi-square test, or the Mann-Whitney $U$ test were used for analysis. The categorical variables were presented as rates and incidences, and assessed by means of the $X^{2}$ test or Fisher's exact test. The log-rank test for Kaplan-Meier time-to-positivity analysis was constructed. Receiver operating characteristic (ROC) curves were plotted for the rate of sensitivity and 1-specificity. Youden's Index was used for optimal cut-off selection, and the area under the curve (AUC) was calculated. The non-parametric test was constructed for the comparison of two groups. Univariate and multivariate logistic regression tests were constructed, for exploring the independent risk factors of septic shock and in-hospital mortality. Univariate logistic regression test was constructed for all independent risk factor exploration of in-hospital mortality, septic shock. Multivariate logistic regression test was constructed for the variates that showed differences in Univariate logistic regression test. The odds ratio (OR) and their 95\% confidence interval (CI) were calculated for the statistically significant variables. All the tests were performed using SPSS (Version 19.0.0 for Windows), and $p$-value $<0.05$ was considered to be statistically significant. 


\section{Results}

\section{Study population}

During the study period, 95 inpatient children with $\geq 1 S$. aureus blood culture positive and systemic inflammation reaction syndrome status were enrolled retrospectively. Of them, 4 cases were lost to follow-up, 3 cases had incomplete clinical information and 4 cases missed their TTPs in medical records. Therefore, 84 cases were finally included in the study (Fig. 1).

\section{Clinical characteristics of $S$. aureus bacteremia in children} The median (IQR) age of the enrolled SAB children was $56.00(10.95,126.25)$ months, median (IQR) weight was $15.00(8.00,35.00)$ kilogram, and $65.5 \%$ (55/84) of them were male. Less than half of the patients $(45.2 \%, 38 / 84)$ had underlying conditions. Among the 35 patients (41.7\%) with prior antibiotic exposure, only 13 cases (15.5\%) reported appropriate. Immunosuppressants were used in 20 cases $(23.8 \%)$. The most frequent infection source was multiple sites $(28.57 \%, 24 / 84)$, followed by pneumonia (27.38\%), and bone or joint infection (15.48\%). Methicillin-resistance was detected in 29 cases (34.5\%), and nosocomial infection in 14 cases (16.7\%).

\section{TTP of S. aureus bacteremia in children}

The median time to positivity (TTP) of blood culture of the 84 SAB children was $21.80 \mathrm{~h}$ (IQR 17.90, 27.42). The bar chart of TTP is shown in Fig. 2. The Kaplan-Meier tests of TTP analysis were constructed respectively with the categorical variables of outcomes. We observed a significant association of TTP with in-hospital mortality $(P=0.001)$ and septic shock incidence $(P=0.001)$ (Fig. 3$)$. Receiver operating characteristic (ROC) curves of the TTP were plotted according to the primary outcome (in-hospital mortality) (See "Clinical outcomes" under Methods). Following Youden's index methodology, we found $16.955 \mathrm{~h}$ to be the optimal cut-off point $(61.5 \%$ sensitivity, $91.5 \%$ specificity, the area under the curves AUC - were 0.776, with 95\% CI 0.625-0.927) (Fig. 4). Therefore, we selected $17 \mathrm{~h}$ as the standard cut-off. Based on this, patients were divided into two groups: early detection group (TTP $\leq 17 \mathrm{~h}$ ) and late detection group (TTP $>17 \mathrm{~h}$ ).

\section{Clinical characteristics and outcomes comparison of early} and late TTP group patients

A comparison between these two TTP groups was shown in Table 1. Demographic characteristics, the proportion of underlying conditions, APACHE II scores, hypoalbuminemia, prior antibiotic exposure, immunosuppressant use, nosocomial infection, methicillin-resistance and length of hospitalization - all were similar between the two groups. However, the other parameters showed important differences. In the early TTP group, $42.9 \%$ cases $(6 / 14)$ needed invasive mechanical ventilation, but only $17.1 \%$ cases $(12 /$

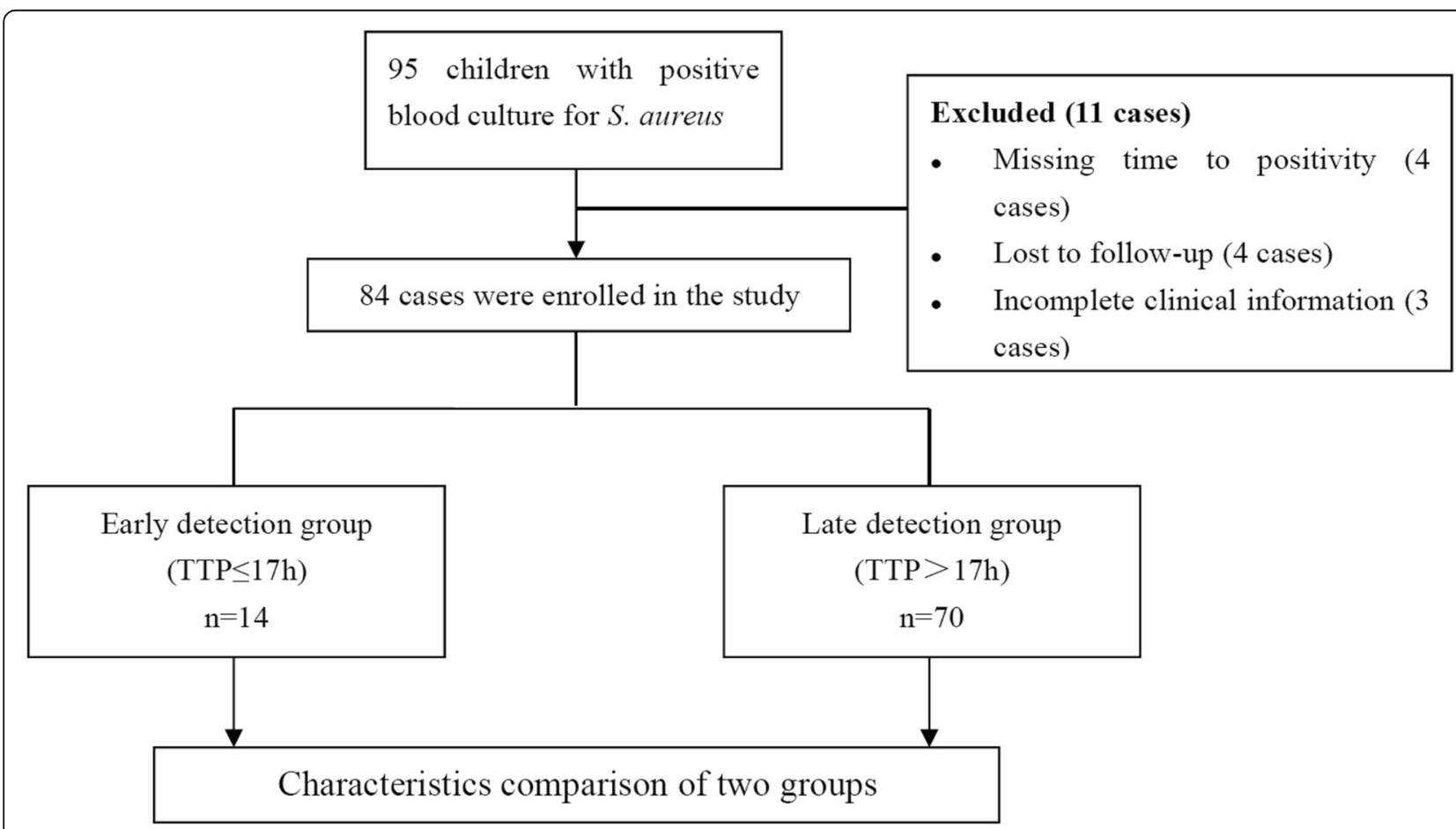

Fig. 1 Flow diagram of the population. As shown, a total of 84 cases were enrolled according to the inclusion and exclusion criteria, and then divided into early detection group (TTP $\leq 17 \mathrm{~h}$ ) and late detection group (TTP $>17 \mathrm{~h}$ ). The clinical characteristics of each group were then determined 


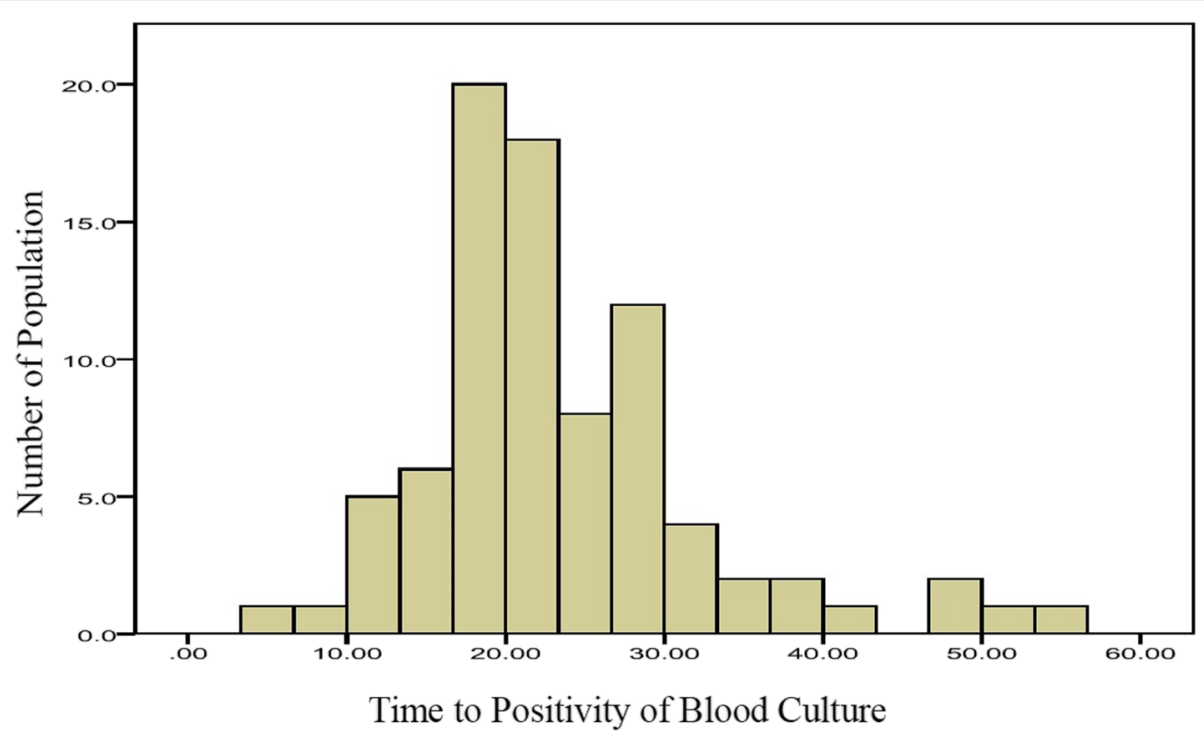

Fig. 2 Bar chart of TTP in Staphylococcus aureus bacteremia children. Out of a total of 84 SAB children, the number of children in each TTP period was plotted against the corresponding TTP, as shown. Details of the quantification have been described in Methods

$70)$ in the late TTP group needed it $(P=0.033)$. Vasoactive agent was needed in $50.0 \%$ cases $(7 / 14)$ in early TTP group but only in $18.6 \%$ cases $(13 / 70)$ in the late TTP group $(P=0.012)$. In-hospital mortality was $57.1 \%(8 / 14)$ in early TTP group but $7.1 \%(5 / 70)$ in late TTP patients $(P=0.000)$. Septic shock occurred in 8 patients $(8 / 14$, $57.1 \%)$ in early TTP group and in 13 patients $(13 / 70$, $18.6 \%)$ in late TTP patients $(P=0.002)$. Taken together, these results indicated critical clinical differences between the early and late TTP groups.

\section{Risk factors of in-hospital mortality}

Univariate logistic regression test demonstrated that in-hospital mortality was correlated with hematologic malignancy, immunosuppressants use, need for invasive mechanical ventilation and vasoactive agent, neutropenia,
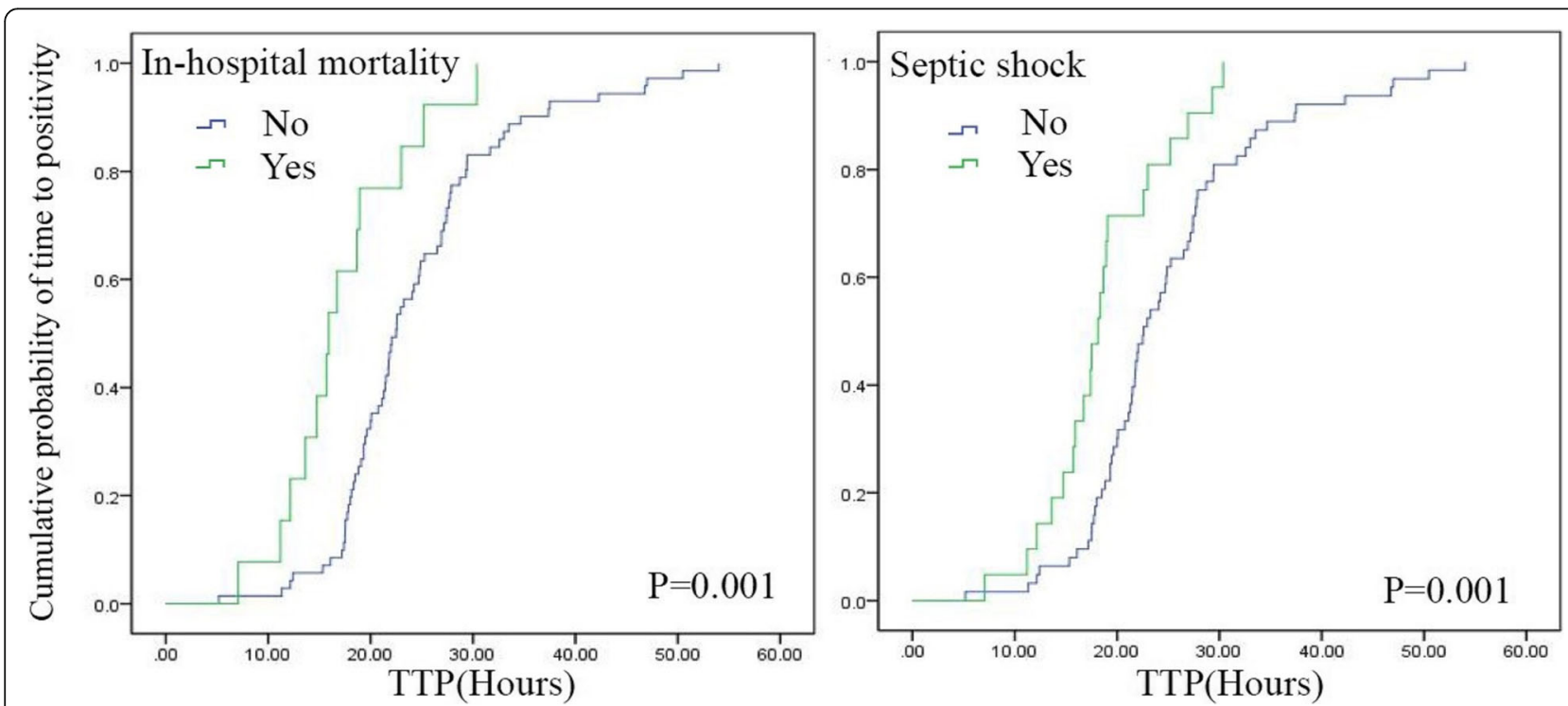

Fig. 3 Comparison of TTP in initial blood culture series according to in-hospital mortality and septic shock. (A) Kaplan-Meier tests were plotted against in-hospital mortality. Cumulative probability of time to positivity of in-hospital mortality patients are presented as the green curve, while survival patients are shown in blue. The difference is significant $(P=0.001)$. (B) Kaplan-Meier tests plotted against septic shock. The green curve represents the cumulative probability of time to positivity of patients with septic shock, and the blue curve represents patients without, the difference being significant $(P=0.001)$ 


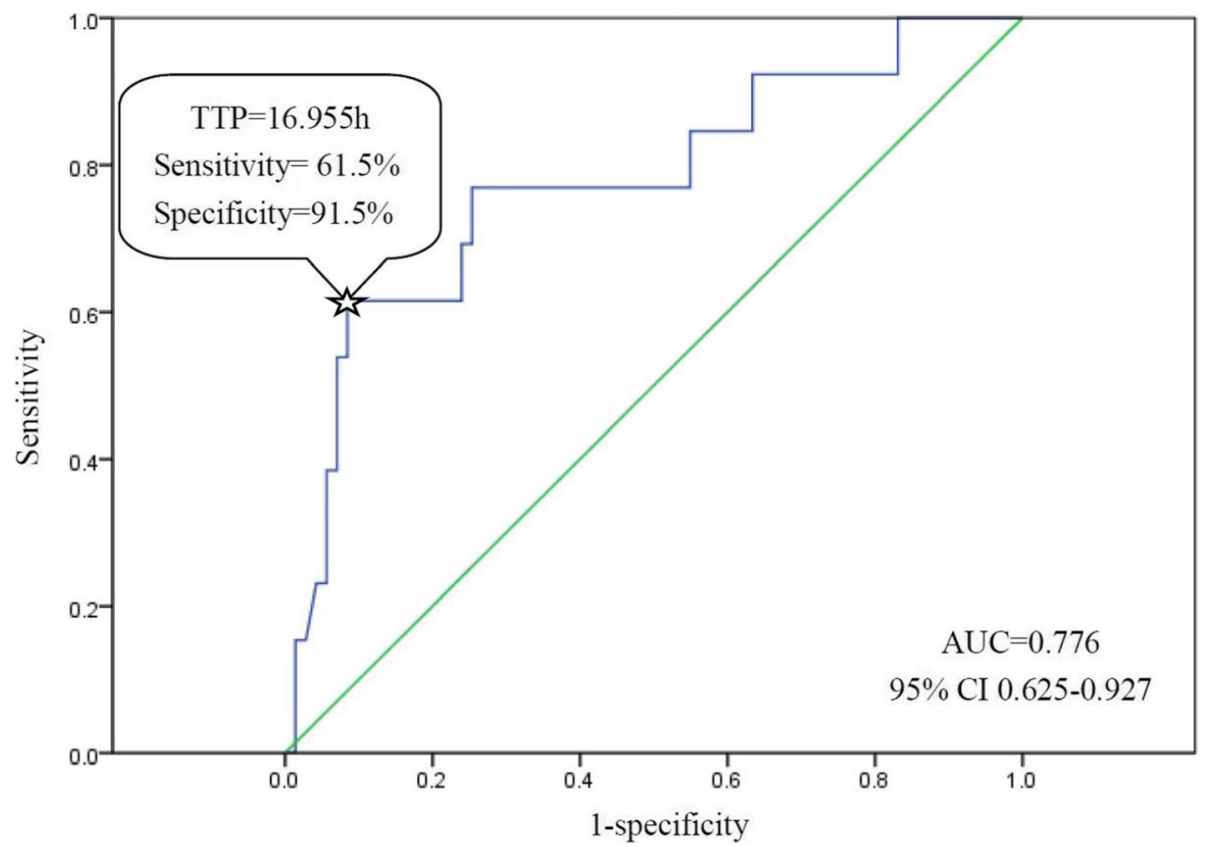

Fig. 4 ROC curves of TTP to predict in-hospital mortality. The ROC curves were plotted according to the in-hospital mortality, and diagonal reference line is also shown. The $X$ axis is 1 -specificity, $Y$ axis is sensitivity, and $16.955 \mathrm{~h}$ was the optimal cut-off point according to Youden's index methodology. As shown above, tis points to the optimal cut-off, sensitivity is 61.5\%, specificity is $91.5 \%$, AUC (area under curve) is 0.776, with $95 \%$ Cl 0.625-0.927

APACHE II score $\geq 15$ and early TTP. Multivariable logistic regression test showed that early TTP, need for vasoactive agent were independent risk factors of in-hospital mortality (Table 2).

\section{Risk factors of septic shock}

The univariate logistic regression test demonstrated that the incidence of septic shock was correlated with hematologic malignancy, immunosuppressants use, need for invasive mechanical ventilation and vasoactive agents, neutropenia, APACHE II score $\geq$ 15, and early TTP. As before, multivariable logistic regression showed that early TTP, need for vasoactive agent and APACHE II score $\geq 15$ were independent risk factors of incidence of septic shock (Table 3).

\section{Discussion}

The prognostic role of TTP in SAB children remained unclear, which prompted us to conduct the present study. We explored the correlation between TTP and clinical characteristics and clinical outcomes in SAB children. As noted above, the median TTP in this group of children was $21.80 \mathrm{~h}$ (IQR 17.90, 27.42), and the optimal TTP cut-off was $16.955 \mathrm{~h}$, which were longer than those of adult $\mathrm{SAB}$ patients having the optimal TTP cut-off of 12 or $14 \mathrm{~h}[10,11,16]$. TTP of blood culture could be influenced by many factors, such as the inoculum, the volume of blood drawn, the incubation conditions and the presence of growth [21]. Although the incubation conditions were stable in our study, only $3 \mathrm{ml}$ blood was extracted to inoculate from our pediatric patients, which was much less than $10 \mathrm{ml}$ in adults. Thus, despite the higher bacterial load in children, this relatively less blood volumes may lead to the long TTPs. Kim et al. [16] stated that the TTP of MRSA was longer than that of MSSA, and nearly one thirds of the bacteria in our study were MRSA, which could also contribute to long TTPs. Our results inferred that adult and childhood SAB patients may present different TTPs; this is consistent with the previous study by Hal SJV et al. [12], which found that age was a predictor of mortality.

To our knowledge, this is the first study to explore the relationship between TTP and clinical outcomes of SAB in children. Here, we have documented that early TTP group patients had 97-fold higher in-hospital mortality and 7-fold higher incidence of septic shock than those with late TTPs. Early TTP was an independent risk factor of in-hospital mortality and septic shock incidence, which indicated that early TTP is indeed associated with worse clinical outcomes in children with SAB. The concept of early TTP of blood cultures is directly related with a higher bacterial load in blood. This is in line with the previous studies in adults, in which early TTP was considered an indicator of poor clinical outcomes [10, $16,17,22,23]$. 
Table 1 Clinical characteristics and outcomes comparison in early and late detection groups

\begin{tabular}{|c|c|c|c|}
\hline Variables & Early Detection Group $(n=14)$ & $\begin{array}{l}\text { Late Detection Group } \\
(n=70)\end{array}$ & $P$ \\
\hline \multicolumn{4}{|l|}{ Demographic characteristics } \\
\hline Age, Median (IQR) & $75.00(16.25-143.50)$ & $49.50(8.49-124.75)$ & 0.275 \\
\hline Weight, Median (IQR) & $23.00(8.50-36.25)$ & $14.50(7.50-32.75)$ & 0.506 \\
\hline Male $(n, \%)$ & $11(78.6 \%)$ & $44(62.86 \%)$ & 0.262 \\
\hline \multicolumn{4}{|l|}{ Underlying Conditions } \\
\hline Hematologic malignancy (n, \%) & $2(14.3 \%)$ & $8(11.43 \%)$ & 0.765 \\
\hline Congenital heart disease $(n, \%)$ & $4(28.6 \%)$ & $10(14.29 \%)$ & 0.193 \\
\hline Neutropenia (n, \%) & $2(14.3 \%)$ & $8(11.43 \%)$ & 0.765 \\
\hline Primary immunodeficiency (n, \%) & $2(14.3 \%)$ & $5(7.14 \%)$ & 0.394 \\
\hline Chronic kidney disease (n, \%) & $1(7.1 \%)$ & $2(2.86 \%)$ & 0.433 \\
\hline \multicolumn{4}{|l|}{ Source of infection } \\
\hline Multiple sites ${ }^{\mathbf{b}}$ & $5(35.7 \%)$ & $19(27.1 \%)$ & 0.519 \\
\hline Pneumonia & $3(21.4 \%)$ & $20(28.6 \%)$ & 0.587 \\
\hline Bone or joint & $0(0.0 \%)$ & $13(18.6 \%)$ & 0.081 \\
\hline Skin or soft tissue & $0(0.0 \%)$ & $9(12.9 \%)$ & 0.158 \\
\hline Primary bacteremia & $0(0.0 \%)$ & $4(5.7 \%)$ & 0.362 \\
\hline Intravenous catheter & $2(14.3 \%)$ & $2(2.9 \%)$ & 0.068 \\
\hline Catheter related infection ${ }^{\mathbf{c}}$ & $1(7.1 \%)$ & $1(1.4 \%)$ & 0.203 \\
\hline Incision & $0(0.0 \%)$ & $2(2.9 \%)$ & 0.525 \\
\hline Endocarditis & $2(14.3 \%)$ & $0(0.0 \%)$ & $0.001^{\mathrm{a}}$ \\
\hline CNS infection & $1(7.1 \%)$ & $0(0.0 \%)$ & $0.025^{\mathrm{a}}$ \\
\hline APACHE II score $\geq 15(n, \%)$ & $5(35.7 \%)$ & $22(31.4 \%)$ & 0.755 \\
\hline Prior antibiotic exposure (n, \%) & $7(50.0 \%)$ & $28(40.0 \%)$ & 0.491 \\
\hline Appropriate antibiotic exposure (n, \%) & $1(7.1 \%)$ & $12(17.1 \%)$ & 0.348 \\
\hline Immunosuppressants use (n, \%) & $6(42.9 \%)$ & $14(20.0 \%)$ & 0.068 \\
\hline Nosocomial infection (n, \%) & $3(21.4 \%)$ & $11(15.7 \%)$ & 0.603 \\
\hline Methicillin-resistance (n, \%) & $4(28.6 \%)$ & $25(35.7 \%)$ & 0.610 \\
\hline Hypoalbuminemia (n, \%) & $1((7.1 \%)$ & $5(7.1 \%)$ & 1.000 \\
\hline \multicolumn{4}{|l|}{ Need for Treatments } \\
\hline Invasive Mechanical ventilation (n, \%) & $6(42.9 \%)$ & $12(17.1 \%)$ & $0.033^{\mathrm{a}}$ \\
\hline Vasoactive agent (n, \%) & $7(50.0 \%)$ & $13(18.6 \%)$ & $0.012^{\mathrm{a}}$ \\
\hline \multicolumn{4}{|l|}{ Outcomes } \\
\hline In-hospital mortality (n, \%) & $8(57.1 \%)$ & $5(7.1 \%)$ & $0.000^{a}$ \\
\hline Septic shock (n, \%) & $8(57.1 \%)$ & $13(18.6 \%)$ & $0.002^{\mathrm{a}}$ \\
\hline $\begin{array}{l}\text { Length of hospitalization } \\
\text { Median (IQR) }\end{array}$ & $17.00(2.75-33.00)$ & $22.50(14.75-37.00)$ & 0.151 \\
\hline
\end{tabular}

${ }^{\mathrm{a}}$ showed difference in the early and late detection groups $(P<0.05)$

${ }^{b} 8$ cases were bone or joint + skin or soft tissue, 5 cases were bone or joint + skin or soft tissue + pneumonia, 4 cases were skin or soft tissue + pneumonia, 5 cases were bone or joint + pneumonia, 2 cases were endocarditis + pneumonia cincluding urethral catheters and thoracic drain tube

We also documented that patients needing vasoactive agents had a 40-fold higher in-hospital mortality and 27 -fold higher incidence of septic shock. Need for vasoactive agent was in fact another independent risk factor of in-hospital mortality and septic shock. Sepsis has been described as a paradigm of acute whole body inflammation with systemic damage in the vascular endothelium [24], and it is well documented that sepsis patients suffered from endothelial disruption and damage [25]. Such damages may result in an 
Table 2 Logistic regression analyses of in-hospital mortality

\begin{tabular}{|c|c|c|c|c|c|c|}
\hline \multirow[t]{2}{*}{ Variables } & \multicolumn{3}{|c|}{ Univariate } & \multicolumn{3}{|c|}{ Multivariate } \\
\hline & $\overline{\mathrm{OR}}$ & $95 \%$ C.I & $P$ & $\overline{\mathrm{OR}}$ & $95 \%$ C.I & $P$ \\
\hline Early TTP & 17.333 & $4.293-69.984$ & 0.000 & 97.494 & $4.477-2123.283$ & 0.004 \\
\hline Need for vasoactive agent & 34.444 & $6.475-183.236$ & 0.000 & 40.513 & $2.988-549.355$ & 0.005 \\
\hline APACHE II score $\geq 15$ & 6.625 & $1.817-24.151$ & 0.004 & - & - & - \\
\hline Neutropenia & 4.815 & $1.136-20.413$ & 0.033 & - & - & - \\
\hline Need for invasive mechanical ventilation & 8.540 & $2.263-32.233$ & 0.002 & - & - & - \\
\hline Immunosuppressants use & 12.273 & $3.208-46.955$ & 0.000 & - & - & - \\
\hline Hematologic malignancy & 4.815 & $1.136-20.413$ & 0.033 & - & - & - \\
\hline
\end{tabular}

impairment of tissue and whole body respiration despite adequate $\mathrm{O}_{2}$ supply. This may also indicate an association with severity and mortality. Not surprisingly, as Badia et al. [26] showed that the need for vasoactive agent is associated with increased risk of mortality in bacteremia patients.

In addition, patients with APACHE II scores $\geq 15$ also presented 10-fold incidence of septic shock, and a statistically significant difference in early TTP group. APACHE II score has been advocated as the gold standard for risk evaluation in critically ill patients [27], and could partly reflect the patient's underlying conditions, translating into unfavorable outcomes [28]. Although APACHE II score has not been used routinely in children these years, some studies demonstrated it can serve as a prognosis factor of children with AKI [29], ARDS [30] and peritonitis [31] effectively. Meanwhile, there were also trends for correlation with hematologic malignancy and neutropenia and immunosuppressant use. These underlying conditions may affect the TTPs and outcomes, consistent with the previous studies $[1,17,22]$. Immune status is also one of the determinants of bacteremia severity [17, 32, 33], and therefore, plays a crucial role in bacteremia patients [22]. Immunosuppressants, widely used in patients with hematologic malignancy, may in fact inhibit the activity of immunocytes [34, 35], leading to neutropenia and decreased bacteria clearance, finally resulting in higher bacterial loads and severe bacteremia.

Our finding that prior antibiotic exposure and appropriate antibiotic exposure had little impact on TTPs and clinical outcomes is consistent with other studies $[16,17,22]$, while differing from those on adults [36, 37]. Nevertheless, van Paridon et al. [38] stated that the timing of antibiotic use in children, presented early use of appropriate antibiotics, had little association with sepsis outcomes. As the details of administration were hardly found in this retrospective sudy, further prospective investigations are needed to determine the effects of appropriate antibiotic exposure on TTPs and clinical outcomes.

Lastly, we would like to address the potential limitations of this study. First, as a single- center study, the data were collected from one academic teaching hospital in China, the results may not easily extrapolate to patients admitted to other hospitals or in other countries. Second, the relatively small sample size of our study $(n=84)$ may result in type II error and reduce the ability to determine the statistical significance of the variables. Third, as the data were collected from the medical records retrospectively, some information was unfortunately missed. Clearly, multi-center, prospective studies in the future, using a larger sample size, should strengthen the results of this study.

Table 3 Logistic regression analyses of septic shock incidence

\begin{tabular}{|c|c|c|c|c|c|c|}
\hline \multirow[t]{2}{*}{ Variables } & \multicolumn{3}{|c|}{ Univariate } & \multicolumn{3}{|c|}{ Multivariate } \\
\hline & $\mathrm{OR}$ & $95 \%$ C.I & $P$ & $\mathrm{OR}$ & $95 \%$ C.I & $\mathrm{P}$ \\
\hline Early TTP & 5.846 & $1.730-19.761$ & 0.004 & 6.757 & $1.094-41.730$ & 0.040 \\
\hline Need for vasoactive agent & 44.250 & $10.571-185.228$ & 0.000 & 27.296 & $5.459-136.488$ & 0.000 \\
\hline APACHE I| score $\geq 15$ & 10.625 & $3.410-33.108$ & 0.000 & 9.821 & $1.885-51.174$ & 0.007 \\
\hline Need for invasive mechanical ventilation & 11.611 & $3.435-39.253$ & 0.000 & - & - & - \\
\hline Neutropenia & 5.900 & $1.475-23.600$ & 0.012 & - & - & - \\
\hline Immunosuppressants use & 9.167 & $2.935-28.629$ & 0.000 & - & - & - \\
\hline Hematologic malignancy & 5.900 & $1.475-23.600$ & 0.012 & - & - & - \\
\hline
\end{tabular}




\section{Conclusion}

Early TTP, need for vasoactive agent were independent risk factors of in-hospital mortality; early TTP, need for vasoactive agent and APACHE II score $\geq 15$ were independent risk factors of septic shock in $S$. aureus bacteremia children. TTP of $\leq 17 \mathrm{~h}$ may correlate with the worse outcomes for these children.

\begin{abstract}
Abbreviations
ANC: Absolute neutrophil count; APACHE II scores: acute physiology and chronic health evaluation II scores; Cl: Confidence interval; CNS: Central nervous system; IQR: Interquartile range; MRSA: Methicillin-resistant Staphylococcus aureus; MSSA: Methicillin-Sensitive Staphylococcus aureus: OR: Odds ratio; ROC: Receiver operating characteristic; SAB: Staphylococcus aureus bacteremia; SIRS: Systemic inflammation reaction syndrome status; TTP: Time to positivity
\end{abstract}

\section{Acknowledgments}

We would like to thank staff the Department of Respiratory Medicine, Children's Hospital of Chongqing Medical University.

\section{Funding}

This work was supported in part by the fund of National Key Clinical specialty (2011-873). The funders had no role in study design, data collection and analysis, decision to publish, or preparation of the manuscript.

\section{Availability of data and materials}

The data-sets analyzed during the current study are available from the corresponding author on reasonable request.

\section{Authors' contributions}

ZXL designed the experiments; YYL performed the experiments and wrote the manuscript; QYL contributed to drawing the figures; $G L Z$ and HM helped in the statistical analyses; YW and QY drew the tables; LL, JW and FTS helped to collect the figures. All authors contributed to manuscript revisions and approved the final version for publication.

\section{Ethics approval and consent to participate}

The study was approved by the Ethics Committee of Children's Hospital of Chongqing Medical University (File No: 201813). All methods were performed in accordance with the relevant guidelines and regulations. All study participants provided written consent for future research.

\section{Consent for publication}

Not applicable.

\section{Competing interests}

The authors declare no financial and non-financial competing interests.

\section{Publisher's Note}

Springer Nature remains neutral with regard to jurisdictional claims in published maps and institutional affiliations.

\begin{abstract}
Author details
${ }^{1}$ Key Laboratory of Pediatrics in Chongqing, Chongqing, China. ${ }^{2}$ Department of Children's Hospital of Chongqing Medical University of Education, Ministry of Education Key Laboratory of Child Development and Disorders, China International Science and Technology Cooperation base of Child development and Critical Disorders, Chongqing, China. ${ }^{3}$ Department of Respiratory Medicine, Children's Hospital of Chongqing Medical University, Chongqing 401122, China.
\end{abstract}

Received: 10 September 2018 Accepted: 15 April 2019 Published online: 17 May 2019

\section{References}

1. Wisplinghoff H, Bischoff T, Tallent SM, Seifert H, Wenzel RP, Edmond MB. Nosocomial bloodstream infections in US hospitals: analysis of 24,179 cases from a prospective nationwide surveillance study. Clin Infect Dis. 2004;39(3): 309-17.

2. Burke RE, Halpern MS, Baron EJ, Kathleen G. Pediatric and neonatal Staphylococcus aureus bacteremia: epidemiology, risk factors, and outcome. Infect Control Hosp Epidemiol. 2009;30(7):636-44.

3. Mylotte JM, Tayara A. Staphylococcus aureus bacteremia: predictors of 30-day mortality in a large cohort. Clin Infect Dis. 2000;31(5):1170-4.

4. Felsenstein S, Bender JM, Sposto R, Gentry M, Takemoto C, Bard JD. Impact of a rapid blood culture assay for gram-positive identification and detection of resistance markers in a pediatric hospital. Arch Pathol Lab Med. 2016; 140(3):267.

5. Frederiksen MS, Espersen F, Frimodt-Møller N, Jensen AG, Larsen AR, Pallesen LV, et al. Changing epidemiology of pediatric Staphylococcus aureus bacteremia in Denmark from 1971 through 2000. Pediatr Infect Dis J. 2007;26(5):398-405.

6. Verhoeven PO, Verhoeven PO, Gagnaire J, Botelhonevers E, Grattard F, Carricajo A, et al. Detection and clinical relevance of Staphylococcus aureus nasal carriage: an update. Expert Rev Anti-Infect Ther. 2014;12(1): $75-89$.

7. Mmc L, Warreman EB, Bernards AT, Veelken JH, Pa VDB, Dekkers OM, et al. Distribution and clinical determinants of time-to-positivity of blood cultures in patients with neutropenia. Eur J Haematol. 2017;100(1):206-14.

8. Perez KK, Olsen RJ, Musick WL, Cernoch PL, Davis JR, Land GA, et al. Integrating rapid pathogen identification and antimicrobial stewardship significantly decreases hospital costs. Arch Pathol Lab Med. 2013;137(9): 1247-54.

9. Ning $Y$, Hu R, Yao G, Bo S. Time to positivity of blood culture and its prognostic value in bloodstream infection. Eur J Clin Microbiol Infect Dis. 2016;35(4):619-24.

10. Khatib R, Riederer K, Saeed S, Johnson LB, Fakih MG, Sharma M, et al. Time to positivity in Staphylococcus aureus bacteremia: possible correlation with the source and outcome of infection. Clin Infect Dis. 2005:41(5):594-8.

11. Lai CC, Wang CY, Liu WL, Hou CC, Huang YT, Hsueh PR. Time to blood culture positivity as a predictor of methicillin resistance in Staphylococcus aureus bacteremia. J Inf Secur. 2011;62(2):190-1.

12. Hal SJV, Jensen SO, Vaska VL, Espedido BA, Paterson DL, Gosbell IB. Predictors of mortality in Staphylococcus aureus bacteremia. Clin Microbiol Rev. 2012;25(2):362.

13. Sullivan TD, Lascolea $L$, Neter E. Relationship between the magnitude of bacteremia in children and the clinical disease. Pediatrics. 1982;69(6):699.

14. Bell LM, Alpert G, Campos JM, Plotkin SA. Routine quantitative blood cultures in children with Haemophilus influenzae or Streptococcus pneumoniae bacteremia. Pediatrics. 1985;76(6):901.

15. Sullivan TD, Jr LSL. Neisseria meningitidis bacteremia in children: quantitation of bacteremia and spontaneous clinical recovery without antibiotic therapy. Pediatrics. 1987;80(1):63-7.

16. Kim J, Gregson DB, Ross T, Laupland KB. Time to blood culture positivity in Staphylococcus aureus bacteremia: association with 30-day mortality. J Inf Secur. 2010;61(3):197-204.

17. Marra AR, Edmond MB, Forbes BA, Wenzel RP, Bearman GM. Time to blood culture positivity as a predictor of clinical outcome of Staphylococcus aureus bloodstream infection. BMC Infect Dis. 2006; 13(1):486

18. Goldstein B, Giroir B, Randolph A. International pediatric sepsis consensus conference: definitions for sepsis and organ dysfunction in pediatrics [J]. Pediatr Crit Care Med. 2005;6(1):2-8.

19. Singer M, Deutschman CS, Seymour CW, Shankar-Hari M, Annane D, Bauer $M$, et al. Developing a new definition and assessing new clinical criteria for septic shock: for the third international consensus definitions for sepsis and septic shock (Sepsis-3). JAMA. 2016;315(8):801-10. https://doi.org/10.1001/ jama.2016.0287.

20. Turnidge JD, Kotsanas D, Munckhof W, Roberts S, Bennett CM, Nimmo GR et al. Staphylococcus aureus bacteraemia: a major cause of mortality in Australia and New Zealand. Med J Aust. 2009;191(7):368-73.

21. Plorde JJ, Tenover FC, Carlson LG. Specimen volume versus yield in the BACTEC blood culture system. J Clin Microbiol. 1985;22(2):292-5.

22. Cillóniz C, Ceccato A, Calle CDL, Gabarrus A, Garcia-Vidal C, Salazar A, et al. Time to blood culture positivity as a predictor of clinical outcomes and severity in adults with bacteremic pneumococcal pneumonia. PLoS One. 2017;12:e0182436 
23. Shi-Ning BO, Jian BO, Ning YZ, Zhao Y, Xiao-Lin LU, Yang JY, et al Relationship between time to positivity of blood culture with clinical characteristics and hospital mortality in patients with Escherichia coli bacteremia. Chin Med J. 2011;124(3):330-4.

24. Szabo C. Chapter 52-Pathophysiological roles of nitric oxide in inflammation. 2000. p. 841-872.

25. Ostrowski SR, Berg RM, Windeløv NA, Meyer MA, Plovsing RR, Møller K, et al. Coagulopathy, catecholamines, and biomarkers of endothelial damage in experimental human endotoxemia and in patients with severe sepsis: a prospective study. J Crit Care. 2013;28(5):586-96.

26. Badia M, Iglesias S, Servia L, Domingo J, Gormaz P, Vilanova J, et al. Mortality predictive factors in patients with urinary sepsis associated to upper urinary tract calculi. Med Int. 2015;39(5):290-7.

27. Corrêa TD, Sanches PR, de Morais LC, Scarin FC, Silva E, Barbas CS. Performance of noninvasive ventilation in acute respiratory failure in critically ill patients: a prospective, observational, cohort study. BMC Pulm Med. 2015:15:144.

28. Brierley J, Carcillo JA, Choong K, Cornell T, Decaen A, Deymann A, et al. Clinical practice parameters for hemodynamic support of pediatric and neonatal septic shock: 2007 update from the American College of Critical Care Medicine. Crit Care Med. 2009;37(2):666-88.

29. Mai X, Zhi HL, Cui RD, et al. The use of APACHE II and ATN-ISI in children acute kidney injury [J]Chinese Journal of Practical Pediatrics; 2011.

30. He YN, Zeng P, Xu J. Predictive value of APACHE II score and PEWS score in predicting the prognosis of children with acute respiratory distress syndrome [J]. Chinese Journal of the Frontiers of Medical Science. 2018; 10(6):161-4.

31. Adesunkanmi A, Oseni SA, Adejuyigbe $O$, et al. Acute generalized peritonitis in african children: assessment of severity of illness using modified APACHE II score [J]. ANZ J Surg. 2015;73(5):275-9.

32. Hill PC, Birch M, Chambers S, Drinkovic D, Ellispegler RB, Everts R, et al. Prospective study of 424 cases of Staphylococcus aureus bacteraemia: determination of factors affecting incidence and mortality. Intern Med J. 2001;31(2):97-103.

33. Kaech C, Elzi L, Sendi P, Frei R, Laifer G, Bassetti S, et al. Course and outcome of Staphylococcus aureus bacteraemia: a retrospective analysis of 308 episodes in a Swiss tertiary-care Centre. Clin Microbiol Infect. 2006;12(4): 345-52

34. Yu S, Yu L. Dexamethasone resisted podocyte injury via stabilizing TRPC6 expression and distribution. Evid Based Complement Alternat Med. 2012; 272:652059.

35. Spies CM, Spies CM, Gaber T, Hahne M, Naumann L, Tripmacher R, et al. Rimexolone inhibits proliferation, cytokine expression and signal transduction of human CD4+ T-cells. Immunol Lett. 2010;131(1):24-32.

36. Kumar A, Roberts D, Wood KE, Light B, Parrillo JE, Sharma S, et al. Duration of hypotension before initiation of effective antimicrobial therapy is the critical determinant of survival in human septic shock. Crit Care Med. 2006; 34(6):1589-96.

37. Kumar A. An alternate pathophysiologic paradigm of sepsis and septic shock: implications for optimizing antimicrobial therapy. Virulence. 2014;5(1): 80-97.

38. Van Paridon BM, Sheppard C, Gg G, Joffe AR. Timing of antibiotics, volume, and vasoactive infusions in children with sepsis admitted to intensive care. Crit Care. 2015;19(1):293

Ready to submit your research? Choose BMC and benefit from:

- fast, convenient online submission

- thorough peer review by experienced researchers in your field

- rapid publication on acceptance

- support for research data, including large and complex data types

- gold Open Access which fosters wider collaboration and increased citations

- maximum visibility for your research: over $100 \mathrm{M}$ website views per year

At $\mathrm{BMC}$, research is always in progress.

Learn more biomedcentral.com/submissions 